\title{
Gerador de sítios de grupos de pesquisa com inclusão automática de conteúdo baseada na plataforma Lattes
}

\author{
Bruno Rego Salomé, Fátima L. S. Nunes, Marcos Lordello Chaim \\ Escola de Artes, Ciências e Humanidades (EACH) \\ Universidade de São Paulo (USP) \\ CEP: 03828-000 - São Paulo, SP - Brasil \\ \{bruno.salome, fatima.nunes, chaim\}@usp.br
}

\begin{abstract}
This paper describes the GPWeb tool whose objective is to facilitate and automate the creation of Web sites of research groups. The GPWeb generates the structure of the Web page including information obtained from the Brazilian National Council for Scientific and Technological Development $(C N P q)$ by accessing its Lattes platform. Moreover, GPWeb allows visualizing information, such as the qualification of the scientific production of the group, according to Qualis classification, created by the High Education Personnel Improvement Coordination (CAPES). In this sense, GPWeb is also a tool to support the research group management and decision making.
\end{abstract}

Resumo. Este trabalho apresenta a ferramenta GPWeb que visa a facilitar e automatizar a criação de sítios de grupos de pesquisa. A GPWeb gera a estrutura da página web preenchendo as seções de conteúdo com informações provenientes da plataforma Lattes, pertencente ao Conselho Nacional de Desenvolvimento Científico e Tecnológico (CNPq). Além disso, permite visualizar informações importantes para auxiliar a tomada de decisões como a classificação Qualis da produção científica do grupo, elaborada pela Coordenação de Aperfeiçoamento de Pessoal de Nível Superior (CAPES).

\section{Introdução}

Os grupos de pesquisa científica são geralmente formados por docentes, pesquisadores e discentes do meio acadêmico cujo objetivo é buscar soluções para problemas da sociedade, inovações tecnológicas e melhorias em rotinas ou situações diversificadas. Uma tarefa importante, portanto, é tornar disponível a informação e o conhecimento gerado pelos grupos de pesquisa científicos brasileiros.

O Conselho Nacional de Desenvolvimento Científico e Tecnológico (CNPq) dispõe de um diretório dos grupos de pesquisa científica em atuação no Brasil. Este diretório disponibiliza informações básicas na rede mundial de computadores - a World Wide Web (Web). No entanto, essa forma de divulgação é limitada.

Uma maneira de produzir sítios Web para divulgação dos grupos de pesquisa é utilizando gerenciadores de conteúdo. Essas ferramentas consistem em plataformas que permitem a exposição de conteúdo variável na Internet, administrado por meio de menus, com estética e navegação configuráveis. Por meio de um painel de controle administrativo, os gerenciadores do sítio podem administrar o conteúdo, gerenciar os 
processos, o acesso e a segurança, bem como customizar e realizar integração com sistemas legados [Moratelli and Valdameri 2002]. Contudo, para ser utilizado, um gerenciador de conteúdo requer um alto nível de conhecimento técnico, principalmente na sua instalação e configuração inicial.

A plataforma Lattes [PLATAFORMA LATTES 2011] é uma arquitetura de informações mantida pelo Conselho Nacional de Desenvolvimento Científico e Tecnológico $(\mathrm{CNPq})$, com a finalidade de gerir suas atividades de fomento e para integrar em um mesmo ambiente os diversos atores ligados ao sistema de inovação do país [Balancieri et al. 2005].

As informações da plataforma Lattes, em conjunto com os recursos dos gerenciadores de conteúdo, podem auxiliar a criação dos sítios de grupos de pesquisa. No entanto, isso requer um sistema prático de instalação e configuração da ferramenta, além de possibilitar uma manutenção de dados mais prática.

Além da questão de disponibilização informação para o público em geral, no contexto atual da administração acadêmica do país, é também importante avaliar a produção científica. A métrica utilizada é o sistema Qualis [WEBQUALIS 2011], administrado pela Coordenação de Aperfeiçoamento de Pessoal de Nível Superior (CAPES) [CAPES 2012]. O Qualis utiliza diferentes formas de classificação de acordo com as diferentes áreas de estudo, qualificando cada publicação segundo os chamados "estratos" (A1, A2, B1, B2, B3, B4, B5, C) sendo A1 o estrato indicativo de qualidade mais elevado e $\mathrm{C}$ o estrato com menor peso [QUALIS PERIÓDICOS 2012].

Neste trabalho é descrita a ferramenta GPWeb (Grupos de Pesquisa na Web) cujo objetivo é gerar sítios Web para grupos de pesquisas utilizando como base a ferramenta de gerenciamento de conteúdo JOOMLA! [JOOMLA!.ORG 2011] e as informações provenientes da plataforma Lattes. Outra característica da GPWeb é permitir a avaliação das produções do grupo de pesquisa segundo a classificação Qualis da área de atuação do grupo.

Para apresentar mais detalhadamente a ferramenta GPWeb, este artigo primeiramente apresenta alguns trabalhos relacionados, na seção 2, comparando-os em características semelhantes à GPWeb e destacando suas diferenças. A seção 3 apresenta um detalhamento técnico da ferramenta. A seção 4 tece uma discussão e disponibiliza resultados a respeito da utilização da ferramenta e, por fim, a seção 5 apresenta as conclusões do artigo e propõe trabalhos futuros para sua continuidade.

\section{Trabalhos relacionados}

$\mathrm{Na}$ literatura alguns trabalhos propõem o uso da plataforma Lattes como fonte de dados para análise de produção científica. Porém, nenhuma delas propõe a criação de sítios dos grupos de pesquisa, conforme pode ser conferido a seguir.

A ferramenta scriptLattes [Mena-Chalco and Cesar-Jr. 2009] propõe a extração e a compilação automáticas de produções bibliográficas, produções técnicas, produções artísticas, orientações, projetos de pesquisa, prêmios e títulos, grafo de colaborações, mapa de geolocalização de um conjunto de pesquisadores cadastrados na plataforma Lattes [SCRIPTLATTES 2011]. Essas informações são disponibilizadas ao usuário por meio de uma página HTML construída automaticamente pela ferramenta. 
O trabalho de Silva (2010) realizou uma análise sobre a integração de redes de colaboração científica e a tecnologia, tendo como foco de estudo a plataforma Lattes. Esta ferramenta teve como base de coleta de dados também a plataforma Lattes, utilizando-os para formar grafos exibindo a rede de colaboração entre os pesquisadores que utilizam a plataforma. Assim como a GPWeb, é uma forma de captura de dados da plataforma Lattes e geração de uma visualização interessante sobre os dados adquiridos.

Algumas principais características que diferenciam a GPWeb das ferramentas apresentadas são: cria páginas HTML que podem ser modificadas pelo gestor do grupo, além de publicá-las automaticamente em seu momento de criação e modificação; fornece possibilidade de avaliar a qualidade das produções científicas de acordo com a métrica Qualis; visa a coletar e relacionar informações de produção bibliográfica de um grupo de pesquisa como um todo, agrupando os dados dos pesquisadores a ele pertencentes.

A ferramenta scriptLattes, que mais se aproxima da proposta da GPWeb, exibe as informações em HTML para os interessados, entretanto não possui informações qualitativas das publicações para disponibilizar dados para análise aos gestores dos grupos de pesquisa, como a GPWeb propõe. Essas informações são de extrema importância para os gestores para que possam realizar a administração dos recursos do grupo de pesquisa de forma concisa e conduzir o trabalho dos pesquisadores de acordo com as expectativas.

As demais ferramentas apresentam características de compilação e disponibilização de informações relativas às publicações ou relações entre pesquisadores; entretanto não realizam uma relação com formas de avaliação das publicações ou publicam as informações em um site.

\section{Descrição da ferramenta GPWeb}

O desenvolvimento do sistema foi realizado em PHP [PHP.NET 2011] uma vez que a ferramenta Joomla! é composta de diversos componentes escritos nessa linguagem. Componentes com funcionalidades similares são agrupados e chamados de módulos, de forma a facilitar a explanação da estrutura do sistema.

A Figura 1 representa o funcionamento do sistema de forma geral, exibindo a interação entre os módulos do gerenciador de conteúdo e as fontes de informações externas. 


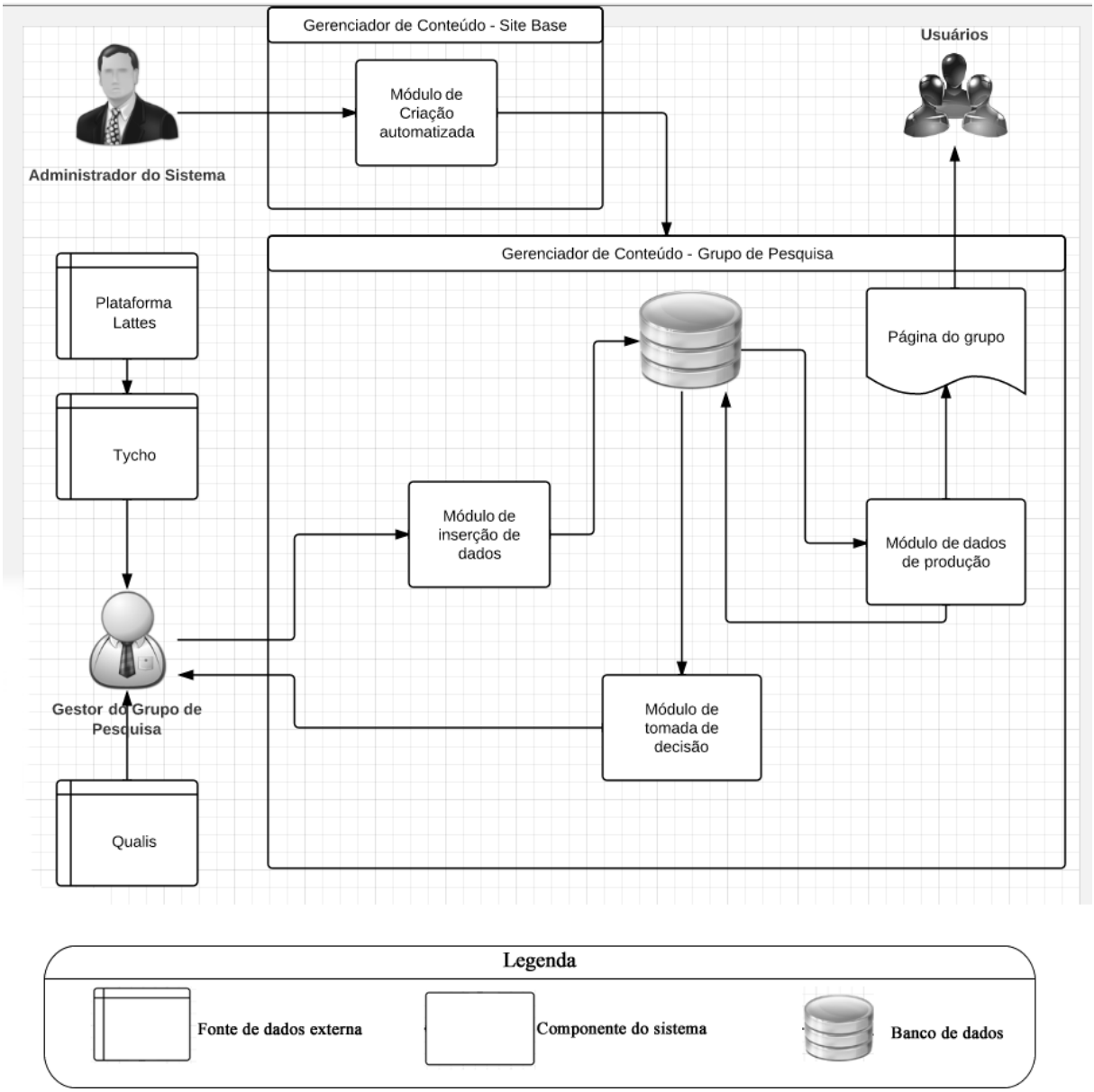

Figura 1. Diagrama de Módulos da Ferramenta GPWeb.

De forma geral, o processo ilustrado na Figura 1, é composto pelas seguintes etapas:

1-) $\mathrm{O}$ administrador da ferramenta GPWeb gera um novo sítio para um grupo de pesquisa;

2-) O gestor do grupo de pesquisa em questão acessa as plataformas Tycho e WebQualis (detalhadas adiante) e obtém os dados necessários para inserção no sistema;

3-) O gestor acessa o site criado e insere os dados coletados por meio do módulo de inserção de dados;

4-) O gestor aciona o módulo de dados de produção para gerar a execução da junção e classificação dos dados;

5-) O gestor acessa o módulo de tomada de decisão para visualizar os resultados.

Em paralelo a todo este processo os usuários acessam o site e visualizam as informações dos grupos de pesquisa. 


\subsection{Módulo de criação automatizada dos sítios}

Este módulo é composto por um componente de criação automatizada de sítios que realiza as operações necessárias via código PHP. Assim é possível que a criação automatizada seja feita por meio um componente do Joomla!.

Os processos para a criação automatizada são:

1-) Copiar a pasta do sítio base no servidor;

2-) Renomear a pasta com o nome do sítio do grupo de pesquisa;

3-) Realizar um backup do banco de dados do sítio base e restaurá-lo em um novo banco com o nome do grupo de pesquisa;

4-) Modificar um arquivo de configuração existente no Joomla! (configuration.php) indicando o novo banco de dados.

O módulo de criação automatizada pode ser visualizado na Figura 2.

Adicionar Grupo

Detalhes

Titulo: lapi|s

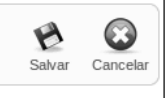

Figura 2. Módulo de criação automatizada de sites.

\subsection{Módulo de inserção de dados}

Este módulo é responsável pela administração da inserção de dados no sistema GPWeb. Exemplos de dados inseridos na GPWeb são os pesquisadores pertencentes ao grupo de pesquisa, as informações do Qualis e os dados provenientes da plataforma Lattes.

Este módulo é dividido em três diferentes componentes, responsáveis pela inserção de cada tipo de dado, como especificado nas subseções a seguir.

\subsubsection{Componente de gerenciamento de pesquisadores}

Este componente é responsável por gerenciar os dados dos pesquisadores que serão utilizados como parâmetro para obtenção de suas publicações.

Ao instalá-lo, o componente cria uma tabela no banco de dados que possuirá os dados de cada pesquisador do grupo e disponibiliza a opção "Administrar Pesquisadores" no menu de componentes do Joomla!.

Por meio deste menu o usuário poderá inserir novos registros na tabela citada anteriormente.

O componente de gerenciamento de pesquisadores pode ser visualizado na Figura 3. 


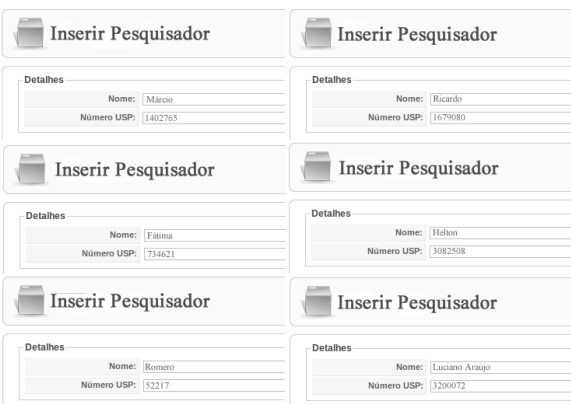

Figura 3. Componente de gerenciamento de pesquisadores.

\subsubsection{Componente de inserção Qualis}

Para executar a classificação dos dados de produção científica, foi utilizado o sistema Qualis que é uma classificação de veículos científicos gerenciada pela CAPES.

A CAPES torna disponível uma ferramenta na Internet [WEBQUALIS 2011] para obtenção de um arquivo em formato Excel com todas as publicações de uma dada área e sua respectiva avaliação. O módulo Qualis da ferramenta GPWeb possui uma interface na qual o usuário pode enviar o arquivo Excel fornecido pela CAPES via um sistema de upload.

O componente realiza a leitura do arquivo e insere as informações existentes nele em uma tabela específica no banco de dados, relacionando um veículo de publicação (com nome, ISSN, ISBN, de acordo com o tipo deste veículo) e seu estrato.

O componente de inserção Qualis pode ser visualizado na Figura 4.

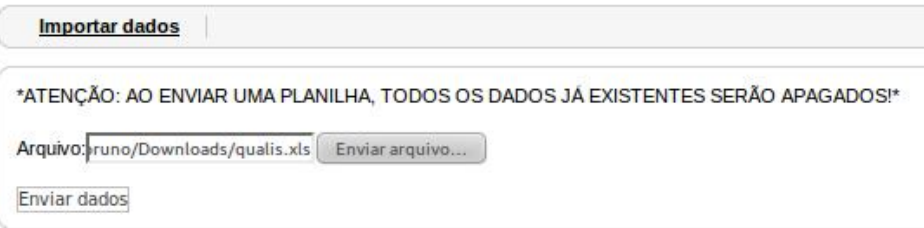

Figura 4. Componente de inserção Qualis.

\subsubsection{Componente de inserção Tycho}

A obtenção dos dados existentes na plataforma Lattes não poderia ser realizada diretamente por acesso ao sítio do $\mathrm{CNPq}$, de forma automatizada, devido a especificidades técnicas impostas pela administração do sítio.

Como forma alternativa de obtenção destes dados foi utilizada a plataforma Tycho [TYCHO, 2011]. Esta plataforma realiza a captura dos dados da plataforma Lattes devido a um acordo formal estabelecido entre a Universidade de São Paulo (USP) e a CAPES. Isso limita as funcionalidades automatizadas atuais da GPWeb apenas a pesquisadores com vínculo com a USP. Assim como a CAPES, a plataforma Tycho oferece um arquivo Excel com as informações solicitadas por meio de interfaces próprias.

Uma forma de contornar a limitação a pesquisadores com vínculo com a USP é inserir as informações adicionais - referentes aos demais pesquisadores participantes do grupo de pesquisa - manualmente em um arquivo Excel no formato aceito pela GPWeb. 
Este procedimento poderia ser adotado por grupos de pesquisa que desejam utilizar a GPWeb e não pertencem à USP.

O componente de inserção de dados do Tycho é composto por um sistema de upload que realiza a leitura deste arquivo Excel e adiciona as informações de publicações científicas presentes neste arquivo em uma tabela do banco de dados.

O componente de inserção Tycho pode ser visualizado na Figura 5.

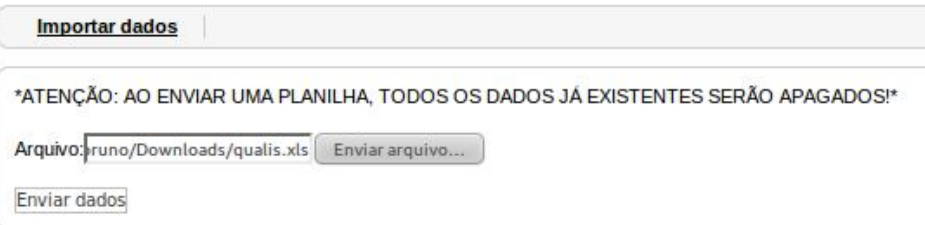

Figura 5. Componente de inserção Tycho.

\subsection{Módulo de dados de produção}

O módulo de dados de produção tem como intuito reunir, organizar e classificar os dados de produção científica dos pesquisadores do grupo de pesquisa presentes no banco de dados.

Ele é composto pelo componente de captura que verifica os pesquisadores cadastrados através do sistema de gerenciamento de pesquisadores. Em seguida, os dados desses pesquisadores, inseridos através do componente de inserção do Tycho, são obtidos.

Ao obter os dados das publicações, o módulo verifica também as informações presentes na tabela com os dados do Qualis e realiza a classificação de cada publicação de acordo com o meio na qual foi vinculada.

O componente realiza essa comparação inicialmente por ISBN. Caso a informação não esteja presente na publicação, o módulo procura por ISSN, e, por fim, por título.

Em seguida todas as informações da publicação, juntamente com sua classificação, são inseridas em uma nova tabela.

O componente também edita algumas informações da publicação, pertinentes à apresentação ao público, e as codifica em formato $H T M L$ para inseri-las à tabela de conteúdo do Joomla!. As informações são inseridas relacionadas à categoria a qual pertence (Livro, Revista ou Evento). Caso a categoria não exista na tabela no Joomla!, o componente a insere na tabela antes de relacioná-la à informação que está sendo inserida.

Após esse processo, as informações estarão disponíveis na página correta no sítio do grupo de pesquisa e as publicações estarão devidamente classificadas no banco de dados do módulo.

\subsection{Módulo de tomada de decisão}

Este módulo é composto pelo componente de relatórios, que oferece uma interface para que o usuário selecione as informações existentes no sistema de acordo com sua necessidade. Ao configurar os filtros desejados, o componente irá disponibilizar uma tabela com as classificações e quantidade de publicações para cada uma delas. Fornece 
também um gráfico de produção anual por estrato, mostrando a quantidade total de publicações para cada classificação por ano, representado pela Figura 6. Além disso, estão disponíveis outros formatos de gráfico (barras verticais, pizza, barras horizontais e linear).

Os gráficos foram desenvolvidos utilizando a ferramenta Google Charts [GOOGLE CHARTS API 2011]. De acordo com a opção desejada selecionada pelo usuário, é criado um link dinamicamente que exibe os gráficos através da API.

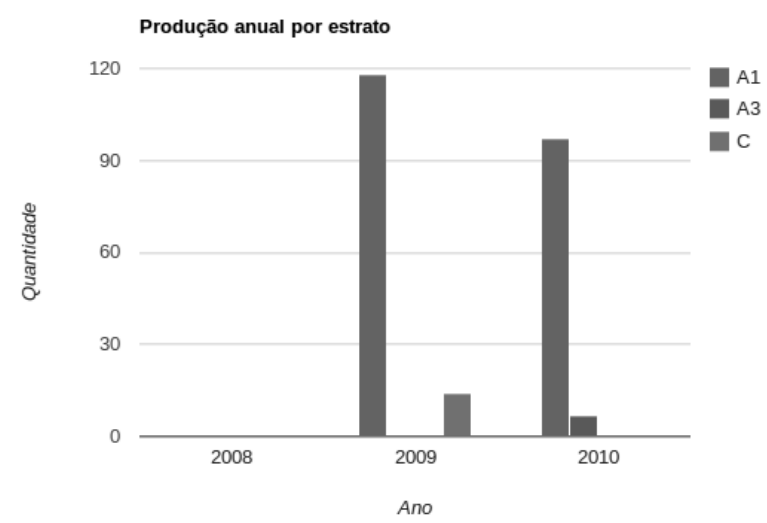

Figura 6. Modelo de gráfico do módulo de tomada de decisão.

\section{Resultados e discussões}

De forma a exemplificar o funcionamento da GPWeb foi realizada a criação do sítio do grupo de pesquisa LApIS (Laboratório de Aplicações de Informática em Saúde), pertencente à Escola de Artes, Ciências e Humanidades da USP.

Para criar o sítio do grupo, utilizou-se o sítio base já existente no servidor e o módulo de criação automatizada do sítio. Em seguida foi inserido o nome do grupo de pesquisa: LApIS. Com isso, o sistema realizou os processos explicados anteriormente para executar uma nova instalação do Joomla! com os dados do grupo LApIS, disponibilizando, assim, um sítio para o grupo de pesquisa, bastando acessá-lo para dar continuidade aos processos (Figura 7).

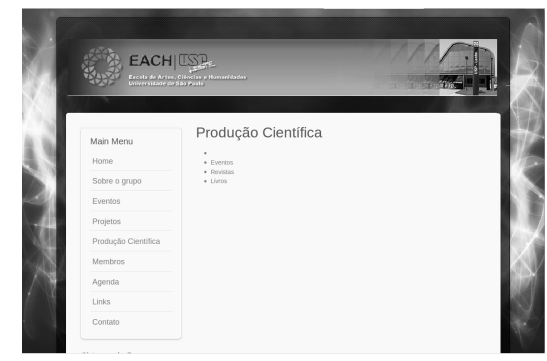

Figura 7. Gráfico de Estrato com Quantidade x Ano.

Com o sítio do grupo de pesquisa já criado, foi executado o módulo de inserção de pesquisadores e as informações dos pesquisadores do grupo foram nele inseridas. Os nomes e números de registro dos pesquisadores são registrados na tabela do banco de 
dados pertinente para que sejam utilizados como parâmetros para pesquisa no módulo de tomada de decisão.

Para que seja possível incluir as informações do Qualis no sistema, primeiramente é necessário obter a planilha de dados oferecida no sítio da CAPES. Para isso basta selecionar, no sítio da CAPES, a área de interesse e gerar o relatório. Para este caso de teste, a área selecionada foi "Ciência da Computação".

Com o intuito de obter informações referentes à qualidade das publicações futuramente, a planilha de dados do Qualis foi enviada ao servidor por meio do módulo de dados de produção, que, em seguida, realizou a leitura desta planilha e inseriu as informações nela presentes em uma tabela do banco de dados.

Para obter os dados necessários a respeito da produção bibliográfica do grupo de pesquisa, foi necessário acessar a plataforma Tycho e filtrar os resultados desejados pelo grupo de Pesquisa. As informações foram inseridas no sistema usando-se do módulo de inserção de dados, realizando-se o upload de arquivos Excel.

Para finalizar o processo, executou-se o módulo de captura de dados, que reúne as informações e as prepara para exibição ao usuário no sítio e no módulo de tomada de decisão, exibindo uma mensagem de confirmação. O módulo de captura realiza uma leitura dos dados de produção bibliográfica existentes no banco de dados e os agrupa em uma única tabela, já os classificando de acordo com as informações do Qualis.

Com isso as informações a respeito das publicações científicas do grupo já foram automaticamente disponibilizadas para os usuários na devida página do sítio (Figura 7).

Por fim, é possível visualizar gráficos que analisam a quantidade de estrato segundo alguns filtros utilizados para realizar a consulta. Os filtros disponibilizados para realizar esta consulta são: Pesquisador, Estrato (Intervalo) e Ano (Intervalo). Desta forma, pode-se pesquisar a produção de um determinado pesquisador (ou de todos), entre um determinado intervalo de estratos (ou de todos) e ainda pelo intervalo de ano de publicação. Como exemplo, foram selecionados todos os pesquisadores e todos os estratos do ano de 2000 até o ano de 2011. Após a inserção de informações desejadas nos filtros, o sistema disponibiliza ao usuário cinco opções diferentes de gráficos. A Figura 8 mostra o resultado o gráfico de quantidade de publicações no período nos diferentes estratos. Na Figura 9 esses dados são mostrados em formato de gráfico de linhas.

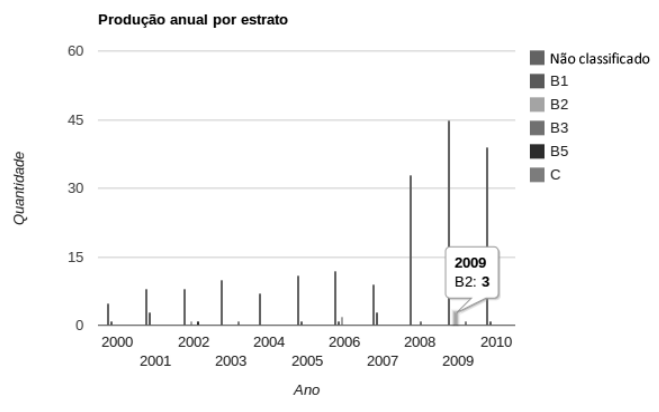

Figura 8. Gráfico de Estrato com Quantidade x Ano. 


\section{Produção por estrato no período}

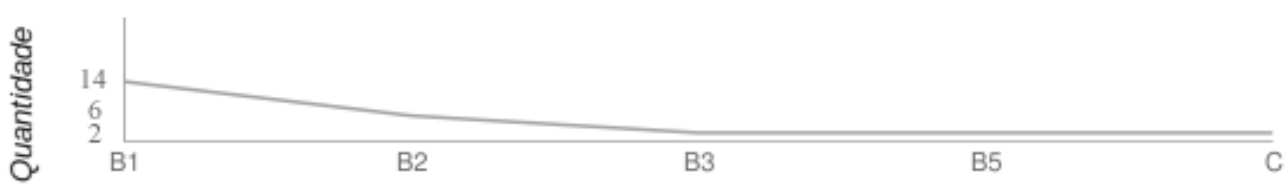

Estrato

Figura 9. Gráfico linear.

Há também duas opções de gráficos de barras: o gráfico de barras horizontais e o gráfico de barras verticais, mostrados nas Figuras 10 e 11, respectivamente. Essas duas opções foram disponibilizadas para atender diferentes necessidades de visualização.

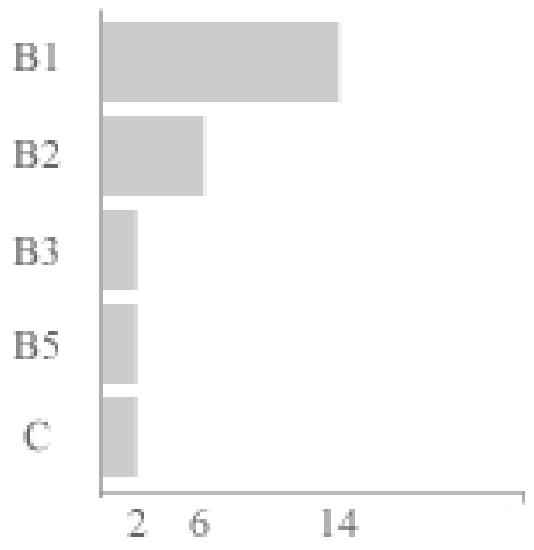

\section{Produção por estrato no período}

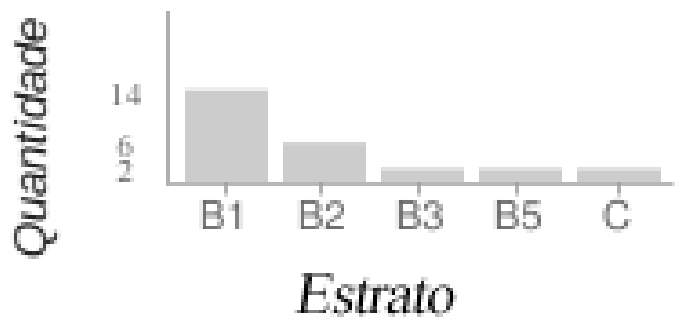

Figura 11. Gráfico de barras verticais.

Figura 10. Gráfico de barras horizontais.

O gráfico no formato de pizza fornece uma visão quantitativa das produções bibliográficas de acordo com seus estratos, no período considerado (Figura 12).

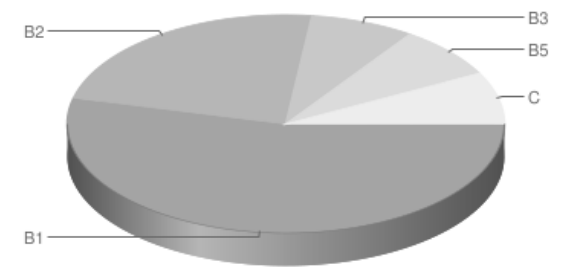

Figura 12. Gráfico de pizza.

Com essas tecnologias agregadas ao Joomla!, o resultado final se apresentou como um produto de fácil manuseio e com fornecimento de informações importantes para se realizar a tomada de decisão, oferecendo diversas opções de visualização.

O desenvolvimento do projeto visou também durante todo o tempo a simplicidade de instalação, deixando os módulos formados por componentes facilmente instaláveis no Joomla!. 


\section{Conclusão}

Neste trabalho foi apresentada a ferramenta GPWeb cujo objetivo é permitir a criação automatizada de sítios de grupos de pesquisa para auxílio à tomada de decisão utilizando dados provenientes da plataforma Lattes.

A ferramenta auxilia os gestores dos grupos de pesquisa a publicar informações na Internet, a administrar informações importantes para o grupo e a analisar a qualidade das publicações a partir da classificação proposta pelo sistema Qualis da CAPES. Além disso, as páginas Web geradas pela GPWeb podem ser customizadas devido às características da plataforma Joomla!, tanto no que diz respeito ao design quanto às funcionalidades disponíveis.

Quanto às limitações do projeto, o principal ponto a salientar é o fato de ainda existir grande quantidade de trabalho manual a ser exercido pelo gestor do grupo de pesquisa. Um exemplo disso é a necessidade de acesso à ferramenta Tycho, para obtenção das planilhas com dados das produções bibliográficas para inserir no sítio, assim como ocorre com os dados do sistema Qualis.

Como trabalhos futuros estão previstas melhorias na interface da GPWeb a fim de oferecer mais opções de edição dos dados e alternativas ao realizar inserção de arquivos Excel, além de gráficos mais elaborados, de acordo com as necessidades apresentadas pelos grupos de pesquisa durante a utilização do sistema.

\section{Agradecimentos}

Os autores agradecem ao Conselho Nacional de Desenvolvimento Científico e Tecnológico (CNPq) - Processo 559931/2010-7, à Fundação de Amparo à Pesquisa do Estado de São Paulo (Fapesp) - Processo 2010/15691-0, ao Instituto Nacional de Ciência e Tecnologia - Medicina Assistida por Computação Científica (INCT-MACC) e à Pró-reitoria de Pesquisa da USP, pelo apoio financeiro.

\section{Referências}

Balancieri, R. et al. (2005), “A análise de redes de colaboração científica sob as novas tecnologias de informação e comunicação: um estudo na Plataforma Lattes", Revista Ciência da Informação, Brasília, v.34, p. 64-77.

CAPES (2012) "CAPES - Coordenação de Aperfeiçoamento de Pessoal de Nível Superior”, http://www.capes.gov.br/ Janeiro (Acessado em: 08 de Janeiro de 2012).

CMS MATRIX (2012) "Compare Content Management Systems - cmsmatrix.org - The Content Management Comparison Tool”, http://cmsmatrix.org/matrix/cms-matrix\# Maio (Acessado em: 26 de Maio de 2012).

Fernandes, L. A. (2005), "Melhoria de Desempenho em Gerenciador de Conteúdo Web usando XML e Banco de Dados", Salvador: Universidade Federal da Bahia.

GOOGLE CHARTS API (2011) "Google Chart Tools - Google Code", http://code.google.com/apis/chart/ Outubro (Acessado em: 24 de Outubro de 2011).

JOOMLA!.ORG (2011) “Joomla!”, http://www.Joomla!.org/ Março (Acessado em: 12 de Março de 2011). 
Mena-Chalco, J.P., Cesar-Jr, R. M. (2009) "scriptLattes: An open-source knowledge extraction system from the Lattes platform", Journal of the Brazilian Computer Society, vol. 15, n. 4 , pp. 31-39.

MICROSOFT EXCEL 2010 (2011) "Microsoft Excel 2010 - Office.com", http://office.microsoft.com/pt-br/excel/ Novembro (Acessado em: 10 de Novembro de 2011).

MICROSOFT WINDOWS (2011) "O que é o Windows 7? - Microsoft Windows.", http://Windows.microsoft.com/pt-BR/Windows7/products/what-is/ Maio (Acessado em: 25 de Maio de 2011).

Moratelli, A. S.; Valdameri, A.R. (2002), "Sistema de gerenciamento de conteúdo para ambiente WEB”, Anais do XI Seminário de Computação, Blumenau, v. 1, n. 1, p. 5364.

PHP.NET (2011) "PHP: Hypertext Preprocessor", http://br.php.net/ Maio (Acessado em: 30 de Maio de 2011).

PLATAFORMA LATTES (2011) "Plataforma Lattes", http://Lattes.cnpq.br/ Março (Acessado em: 12 de Março de 2011).

QUALIS PERIÓDICOS (2012) “WebQualis”, http://www.capes.gov.br/avaliacao/qualis/ Janeiro (Acessado em: 08 de Janeiro de 2012).

REGISTRO.BR (2011) "Registro.br", http://registro.br/ Março (Acessado em: 12 de Março de 2011).

SCRIPTLATTES (2011) "scriptLattes", http://scriptlattes.sourceforge.net/ Fevereiro (Acessado em: 09 de Fevereiro de 2011).

Silva, E. E. (2010), "Estudo e Desenvolvimento de Ferramentas para a Análise de Redes

Sociais de Pesquisa”, São Paulo: EACH-USP, p. 48.

TYCHO (2011) “Tycho”, https://sistemas.usp.br/tycho/start.jsp/ Outubro (Acessado em: 24 de Outubro de 2011).

WEBQUALIS (2011) "WebQualis", http://qualis.capes.gov.br/webqualis/ Outubro (Acessado em: 24 de Outubro de 2011).

Yamaoka E. J. (2006), "O uso da Internet. Métodos e técnicas de pesquisa em comunicação", v.2, p. 146-162. 